\title{
Incidence of Renal Tract Abnormalities on Ultrasonography in Patients with Spinal Cord Injury: A Retrospective Pilot Study of a Military Cohort Undergoing Long-Term Institutional Rehabilitation
}

\author{
Mandeep Saini ${ }^{1}$, Mohit Kataruka ${ }^{2}$, Biraj Gogoi ${ }^{3}$, Vyom Sharma ${ }^{3}$, Gurdarshdeep Singh Madan ${ }^{1}$, Chetan Sood ${ }^{3}$ \\ ${ }^{1}$ Department of Radiodiagnosis and Imaging, Military Hospital Kirkee, Pune, India \\ ${ }^{2}$ Department of PMR, Spinal Cord Injury Centre, Military Hospital Kirkee, Pune, India \\ ${ }^{3}$ Department of Orthopaedics and Spine Surgery, Spinal Cord Injury Centre, Military Hospital Kirkee, Pune, India
}

\section{Study Design: Retrospective pilot study.}

Purpose: To assess the incidence of renal tract abnormalities using ultrasonography (US) in a military cohort with traumatic spinal cord injury (TSCI) at a tertiary level spinal cord injury center.

Overview of Literature: Neurogenic bladder in TSCI patients results in significant urological morbidity. There is lack of data for these patients during the first 18 months of long-term rehabilitation in an institutional setting.

Methods: We retrospectively reviewed patient records to collect data on demographic characteristics, injury level, injury severity, time since injury, bladder management methods (such as an indwelling catheter [IC], clean intermittent catheterization [CIC], or selfvoiding [S]); we correlated these data with the findings of the renal tract US.

Results: The study included 73 out of 81 male participants. The mean patient age was 29.99 years; the study group included $34.2 \%$ tetraplegics and $65.8 \%$ people with paraplegia. The time since injury was 6-12 months for $42.5 \%$ of the subjects and $12-18$ months for $57.5 \%$ of the subjects. A normal US scan was recorded in $65.7 \%$ patients, and bladder trabeculation was the commonest finding in $15.1 \%$ of the subjects, followed by hydronephrosis (HDN) in $12.3 \%$, and renal calculus and atrophy in $1.3 \%$ participants each. We found $22.22 \%$ of the IC group participants had higher US abnormalities than those in the reflex voiding group (statistically nonsignificant difference, $p=0.7)$. Trabeculations $(21.4 \%)$ and HDN (19\%) were more common in those who had sustained the injury 12-18 months previously as compared to that in those who had injured themselves $6-12$ months previously $(p=0.04)$. The proportion of patients who had a normal US scan was higher in the group who sustained the injury 6-12 months previously versus those who had sustained the injury 12-18 months previously; the difference was statistically significant $(p=0.02)$. There was no significant $(p=0.72)$ correlation in the bladder management method, injury level, and renal tract abnormalities between the groups.

Conclusions: This retrospective study shows that $65 \%$ of TSCI participants had no renal tract abnormality on US scan and bladder trabeculation ruled out as the most common finding. Long-term supervised rehabilitation may help achieve good renal quality of life; however, further prospective trials are required on this subject.

Keywords: Spinal cord injuries; Renal tract ultrasonography; Institutional rehabilitation; Neurogenic bladder; Military soldier

Received Jan 31, 2021; Revised Jan 31, 2021; Accepted Mar 11, 2021

Corresponding author: Vyom Sharma

Department of Orthopaedics and Spine Surgery, Spinal Cord Injury Centre, Military Hospital Kirkee, Pune, India

Tel: +91-20-25818705, Fax: +91-20-25818704, E-mail: vyomsharma.479p@gov.in 


\section{Introduction}

Traumatic spinal cord injury (TSCI) is sustained by about 250,000 to 500,000 persons globally every year as per World Health Organization; there are about 15 new cases per million annually in India [1], and this injury represents a life-changing event for an individual. The chronic phase following TSCI involves potential complications spanning multiple organ systems; renal tract dysfunction and neurogenic bladder are the leading causes of such complications [2]. In the earlier part of the 20th century, the two common sequelae of neurogenic bladder, namely urosepsis and renal failure, had been attributed as causes of death in up to $35 \%$ of TSCI patients [3,4]. The evolution and progress in such individuals' care, reflected by the statistics on higher survival and better life expectancy $[5,6]$, also reduce mortality due to urological infections [7]. However, neurogenic bladder sequelae can result in significant morbidity because of urinary tract infections (UTIs), calculus disease, hydronephrosis (HDN), bladder carcinoma, renal failure, and morphological alterations of the urethra and the bladder [8]. Therefore, an efficient and effective bladder or urological routine and urinary surveillance will determine whether a TSCI patient would develop urological complications [9]. An effective bladder routine encompasses the patient and the caregiver's training regarding the adoption of an appropriate bladder drainage method and maintenance of a healthy urological quality of life (QOL). However, despite an effective and good bladder management routine using any of the methods, such as an indwelling catheter (IC), clean intermittent catheterization (CIC), and self-voiding (S), urological morbidity continues to be associated with chronic TSCI and indicate the importance of urological surveillance. The method and timing of urological surveillance have been debated in several studies recommending ultrasonography (US) of the renal tract as a safe, affordable, and cost-effective tool for routine surveillance method for detecting renal tract complications $[10,11]$. There is a lack of data on urological morbidity in TSCI patients undergoing long-term, supervised rehabilitation in an institutionalized setting where patients from a military cohort were enrolled in the rehabilitation program for 18-24 months. We designed our study to assess the incidence of abnormalities in the renal tract detected during routine US surveillance and correlation of bladder management routine and time from injury at the largest, tertiary level spinal cord injury (SCI) center of the Indian Armed Forces.

\section{Materials and Methods}

\section{Patients}

The ethics committee of Armed Forces Medical College, Pune, India approved this retrospective, cross-sectional pilot study (IRB approval no., IEC/2020/188). In view of the study design being retrospective, informed consent was in the study protocol. The study was conducted at a tertiary care Armed Forces rehabilitation center for SCI. Medical and radiological records of all the patients admitted to the SCI center between January 2018 and December 2019. As per the inclusion criteria, the study enrolled patients with post-traumatic tetraplegia and paraplegia, aged $>18$ and $<60$ years, with duration of injury $>6$ months. The study excluded patients with a known history of renal disease, frequent UTIs, non-traumatic SCI, duration of injury $<6$ months, and those who had not undergone a US examination of the renal tract. We collected the study participants' demographic data, such as age, etiology, time since injury, neurological level of injury, and American Spinal Cord Injury Association score as per the International Standards for Neurological Classification of Spinal Cord Injury from the existing records [12]. The participants' history regarding the bladder management protocol being followed was obtained. The tetraplegic and paraplegic patients were divided into the following three groups as per their bladder management routine that involved using an IC, CIC, or S via abdominal pressure or reflex (S).

The renal tract's US examination was performed as per the renal surveillance protocol by an experienced senior radiologist using a high-end color Doppler machine (Wipro GE Logiq P5; GE Healthcare, Chicago, IL, USA). The findings of US examination for each subject were recorded in broad categories, such as normal scan, trabeculated bladder, renal calculus, bladder calculus, renal cysts, HDN, and hydroureteronephrosis. The findings of post-void residual urine (PVRU) were recorded for each study subject.

\section{Data analysis}

The findings of the US examination of the renal tract were correlated with the bladder management routine, injury level, time since injury, and PVRU. The data were analyzed using IBM SPSS ver. 20.0 (IBM Corp., Armonk, NY, 
USA), and the $p$-value for statistical significance was kept at $<0.05$. We used the chi-square test for the correlation analysis.

\section{Results}

The study enrolled 81 patients; of these, 73 met the inclusion criteria. Table 1 shows the demographic characteristics of the study subjects. Only male patients with a mean age of 29.99 years (standard deviation $=8.08$ ) comprised the study group; $65.8 \%$ had paraplegia, while 58.9 had complete injuries. The time since injury was $>12$ months for $57.5 \%$ of the patients and 6 to 12 months for $42.5 \%$ of the patients.

\section{Bladder management routine}

Total $57.5 \%$ of the subjects used the S method of blad-

Table 1. Demographic characteristics of study participants

\begin{tabular}{|c|c|}
\hline Characteristic & Value \\
\hline Age (yr) & $29.99 \pm 8.08$ \\
\hline \multicolumn{2}{|l|}{ Time from injury (mo) } \\
\hline $6-12$ & $31(42.5)$ \\
\hline$>12-18$ & $42(57.5)$ \\
\hline \multicolumn{2}{|l|}{ Level of spinal cord injury } \\
\hline Tetraplegia & $25(34.2)$ \\
\hline Paraplegia & $48(65.8)$ \\
\hline \multicolumn{2}{|l|}{ Severity of injury } \\
\hline Incomplete & $30(41.1)$ \\
\hline Complete & $43(58.9)$ \\
\hline \multicolumn{2}{|l|}{ Bladder management } \\
\hline Self-voiding & $42(57.5)$ \\
\hline Clean intermittent catheterization & $22(30.1)$ \\
\hline Indwelling catheter & $9(12.3)$ \\
\hline
\end{tabular}

Values are presented as mean \pm standard deviation or number (\%).

Table 2. Bladder management method as per level of injury al

\begin{tabular}{lccc} 
Variable & Total & Tetraplegia & Paraplegia \\
\hline Indwelling catheter & $9(12.3)$ & $4(44.4)$ & $5(55.5)$ \\
Clean intermittent catheterization & $22(30.1)$ & $6(27.2)$ & $16(72.7)$ \\
\hline Self-voiding & $42(57.5)$ & $14(33.3)$ & $28(66.6)$ \\
\hline
\end{tabular}

Values are presented as number (\%).

${ }^{a}$ No significant correlation $(p=0.64)$. der management, followed by $30.1 \%$ who used CIC, and $12.3 \%$ who used an IC. Table 2 shows the pattern of bladder management routine among tetraplegic and paraplegic study subjects. Among participants who used S either with Crede's method or abdominal tapping (S), 33.3\% were tetraplegics, while $66.6 \%$ of paraplegic subjects could void urine using this method. The bladder management method was not significantly correlated with the injury level $(p=0.64)$.

\section{Ultrasonography findings}

Fig. 1 shows US findings of the renal tract of all the participants. The subjects underwent US examinations every 6 months; $65.8 \%$ had a normal scan, while $15.1 \%$ were detected with bladder trabeculation followed by HDN in $12.3 \%$. Renal calculus and renal atrophy were observed in $1.3 \%$ participants each; $2.7 \%$ of the subjects had renal cysts associated with features of chronic cystitis, and the bladder diverticulum was detected in 1 (1.3\%) of the subjects was labeled as "others".

\section{Ultrasonography findings as per level and severity of injury}

Table 3 shows the correlation of US findings with the level of injury. The majority of normal US scans were observed in $66.6 \%$ paraplegics, while a higher proportion of $64 \%$ tetraplegics and $76.6 \%$ with incomplete injuries had a normal US scan. Similarly, the trabeculated bladder finding was higher in tetraplegics and those with incomplete injuries (16\% and $16.6 \%$, respectively) compared to $14.5 \%$ in paraplegics and $13.9 \%$ with complete injuries. A higher proportion of HDN was observed in $14.5 \%$ of paraplegics and $16.2 \%$ participants with complete injuries

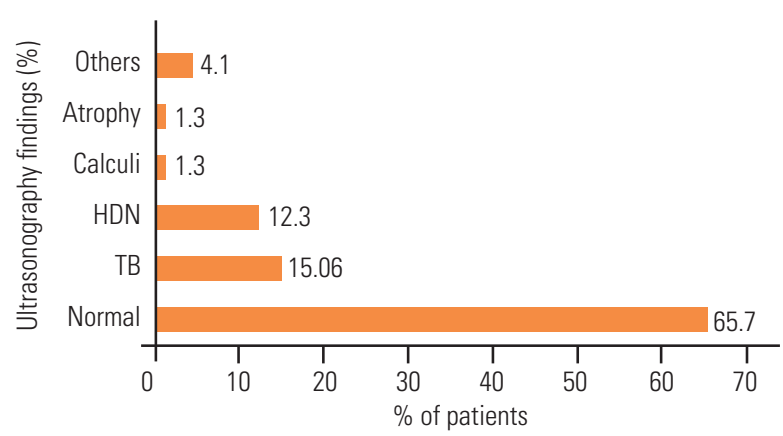

Fig. 1. Renal tract ultrasonography findings in study participants. HDN, hydronephrosis; TB, bladder trabeculation. 
Table 3. Ultrasonography examination findings as per level and severity of injury

\begin{tabular}{lllllll}
$\begin{array}{l}\text { Variable } \\
\text { Level of spinal cord injury }\end{array}$ & Total & Normal & TB & HDN & Calculi & Atrophy \\
$\begin{array}{l}\text { Paraplegic } \\
\text { Tetraplegic }\end{array}$ & $48(65.8)$ & $32(66.6)$ & $7(14.5)$ & $7(14.5)$ & 0 & 0 \\
\hline $\begin{array}{l}\text { Severity of injury } \\
\text { Complete }\end{array}$ & $25(34.2)$ & $16(64.0)$ & $4(16.0)$ & $2(8.0)$ & $1(4.0)$ & $1(4.0)$ \\
\hline Incomplete & $43(58.9)$ & $25(58.1)$ & $6(13.9)$ & $7(16.2)$ & $1(2.3)$ & $1(2.3)$ \\
\hline
\end{tabular}

Values are presented as number (\%).

TB, bladder trabeculation; HDN, hydronephrosis.

Table 4. Ultrasonography findings in different bladder management groups ${ }^{\text {a) }}$

\begin{tabular}{|c|c|c|c|c|c|c|}
\hline Variable & Total & Normal & Atrophy & Calculi & $\mathrm{TB}$ & Others \\
\hline Clean intermittent catheterization & $22(30.1)$ & $14(63.6)$ & $1(4.5)$ & 0 & $2(9.09)$ & $1(4.5)$ \\
\hline Indwelling catheter & $9(12.3)$ & $4(44.4)$ & 0 & $1(11.1)$ & $2(22.2)$ & $1(11.1)$ \\
\hline Self-voiding & 42 (57.5) & $30(71.4)$ & 0 & 0 & $7(16.6)$ & $1(2.3)$ \\
\hline
\end{tabular}

Values are presented as number (\%).

$\mathrm{TB}$, bladder trabeculation.

a) No significant correlation $(p=0.7)$.

as compared to $8 \%$ tetraplegics and in $6.6 \%$ of those with incomplete injuries. Renal calculus and atrophy findings were observed in people with tetraplegia with complete injuries. There was no significant correlation of the level and severity of injury with the US findings ( $p=0.92$ and $p=0.71$, respectively).

\section{Ultrasonography findings as per the bladder manage- ment routine}

Table 4 shows that $71.4 \%$ of the subjects who used $\mathrm{S}$ had no abnormal findings in the US, followed by $63.6 \%$ of those in the CIC group. There was a proportionately higher incidence of abnormal findings (22.2\%) in those with an ICI compared to that in $16.6 \%$ of those with S; both these subsets showed trabeculated bladder wall on the US. There was no significant correlation of the bladder management method with the US findings ( $p=0.7$ ).

\section{Correlation of ultrasonography findings with time since injury}

The analysis of US findings for subjects with time since injury 6-12 months and those with time since injury $12-18$ months, as shown in Table 5. Trabeculated bladder and
Table 5. Ultrasonography findings following injury and correlation with time since injury ${ }^{\text {al }}$

\begin{tabular}{|lcccccccc|}
\hline Time & Total & Normal & Atrophy & HDN & Calculi & TB & Others \\
\hline $6-12$ mo & $31(42.5)$ & $27(87.09)$ & 0 & $1(3.2)$ & 0 & $2(6.4)$ & $1(3.2)$ \\
\hline $12-18$ mo & $42(57.5)$ & $21(50.0)$ & $1(2.3)$ & $8(19.0)$ & $1(2.3)$ & $9(21.4)$ & $2(4.7)$ \\
\hline
\end{tabular}

Values are presented as number (\%).

HDN, hydronephrosis; TB, bladder trabeculation.

a) Statistically significant $(p=0.046)$.

Table 6. Hydronephrosis in patients with different bladder management methods and post-void residual urine $>100 \mathrm{~mL}$ and as per level of injury ${ }^{\mathrm{al}}$

\begin{tabular}{lcc} 
Variable & Paraplegic & Tetraplegic \\
\hline Indwelling catheter & $1(14.2)$ & 0 \\
CIC/CISC & $2(28.5)$ & $1(50.0)$ \\
Self-voiding & $4(57.1)$ & $1(50.0)$ \\
Total & 7 & 2 \\
\hline
\end{tabular}

Values are presented as number (\%).

$\mathrm{CIC/CISC}$, clean intermittent catheterization/clean intermittent self-catheterization.

${ }^{\text {a) }}$ No significant correlation $(p=0.72)$.

HDN were more prevalent in the $12-18$ months group than in the $6-12$ months group $(21.4 \%$ and $19 \%$ versus $6.4 \%$ and $3.2 \%$, respectively; $p=0.04$ ). There was a significant difference in the proportion of participants with a 


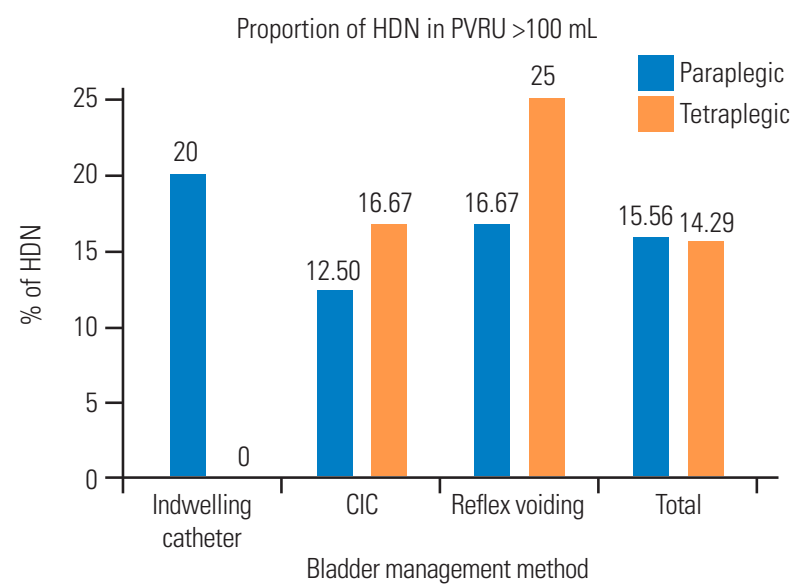

Fig. 2. Finding of hydronephrosis (HDN) correlated with bladder management and post-void residual urine (PVRU) $>100 \mathrm{~mL}$. CIC, clean intermittent catheterization.

normal US scan in the 6-12 months and 12-18 months group $(87.09 \%$ versus $50 \%$, respectively; $p=0.02)$.

\section{Correlation of hydronephrosis and post-void residual urine $>100 \mathrm{~mL}$}

Table 6 shows the correlation analysis of the nine participants who had HDN on US with a PVRU $>100 \mathrm{~mL}$. HDN was detected in tetraplegics and paraplegics who used S, as shown in Fig. 2. In contrast, subjects who used CIC had a lower incidence of hydronephrotic and hydroureteronephrotic changes than those with S. There was no significant correlation between the bladder management method and injury level $(p=0.72)$.

\section{Discussion}

Whether normal micturition and urinary function can be achieved following SCI is debatable. Many develop a neurogenic bladder eventually [9]. Therefore, these subjects' urological vulnerability merits meticulous screening because most urinary tract abnormalities remain undetected $[11,13]$. TSCI patients experience urological instability imposed upon by the spinal shock initially and subsequent period until they adopt a particular urinary drainage method. There are reports on a higher incidence of urinary tract abnormalities during the first year postTSCI, especially the urinary calculi $[14,15]$. Our study aimed to document the incidence of abnormal findings on surveillance US examination because our study population comprised patients who had sustained an injury within the previous 2 years. Moreover, we aimed to determine whether a supervised rehabilitation regime in our cohort effectively reduced the number of abnormalities detected on renal tract US.

In our study, $18.1 \%$ of the patients had trabeculated bladder within a year of TSCI, and $81.8 \%$ had trabeculated bladder 12-18 months after the injury. This is in contrast to the findings reported by Hoffberg and Cardenas [16] in 1986 and Güzelküçük et al. [10], who reported an overall prevalence of $57.1 \%$ and $35.1 \%$ and a 1 -year post-injury prevalence of $31 \%$ and $27.2 \%$, respectively. The trabeculated bladder can result from high detrusor pressure and structural deterioration of the bladder wall that can manifest within the first year after a TSCI $[17,18]$. The clinical significance of trabeculated bladder has been highlighted by Güzelküçük et al. [10] because bladder trabeculation can be one of the earliest signs of a developing renal tract abnormality, such as calculi and renal atrophy, in TSCI patients. Our study subjects who used S had the highest proportion of trabeculated bladder (63.6\%); thus, there may be a considerable contribution of high abdominal pressure used during the reflex voiding maneuvers [19]. However, the hypothesis of high abdominal pressure contributing to a higher incidence of trabeculated bladder in our study is not supported by a lower incidence of HDN and a non-significant correlation between them. There is a significant correlation between time since the injury of 12-18 months and trabeculated bladder in our cohort $(p=0.04)$, although we did not observe the same in most of our patients within the first year of TSCI that can be attributed to a good bladder management routine, though not statistically significant $(p=0.7)$. The traditional analysis of time since injury in earlier studies regarding renal abnormalities in TSCI patients has focused on the first year after the injury and the subsequent years [10]; in contrast, in our study, the patients were divided as per those who had sustained an injury 6-12 months previously and those who had injured themselves 12-18 years previously.

HDN was detected in $12.3 \%$ of patients overall and in $11.1 \%$ during the first year after the injury. Gormley [8] and subsequent researchers have shown the role of poor bladder compliance, renal calculus disease, and detrusor dysfunction and the significance of early detection of HDN to avoid the catastrophic eventuality, such as renal insufficiency and renal failure [20,21]. Our results show a significant correlation of a higher proportion of HDN 
at 12-18 months post-TSCI. The analysis of the pattern of HDN in our study patients, whose PVRU was $>100$ $\mathrm{mL}$, showed a higher prevalence of $\mathrm{HDN}$ in patients with $\mathrm{S}$ and the lowest prevalence in the CIC group. This confirmed CIC's positive effect in maintaining optimum renal function, as shown by Pettersson-Hammerstad et al. [22] in their retrospective review on improvement in renal function with $\mathrm{CIC}$ in such patients. The role of renal tract calculi-inducing HDN in our cohort was unclear because only one study subject had a renal calculus. Our study's findings showed a deviation from the expected trend of a higher proportion of renal or bladder calculi, as reported earlier $[10,11,23]$. Our results did not demonstrate any renal tract calculi in the 6-12 months group in contrast to the landmark study by Chen et al. [24], reporting a higher risk of bladder and renal calculi after SCI within the first 3 months of injury that gradually declined over the subsequent years. We attribute this finding in our study to a good bladder management routine that helped avoid the probable risk factors for renal tract calculi, such as increased intravesical pressure and avoidance of hypercalciuria in our ambulant patients [25].

The association of renal tract abnormalities with the level and severity of injury indicated a higher incidence of HDN in patients with complete injuries and tetraplegics, while trabeculated bladder was more common in paraplegics with incomplete injuries. This observation is consistent with findings by earlier investigators that show a higher incidence of renal calculi in high level and complete injuries [10] and a significant effect of suprasacral lesions to manifest with detrusor hyperreflexia and/or detrusor sphincter dyssynergia, leading to calculi as well as HDN [26]. All our subjects had undergone surveillance US examination by the same senior radiologist (first author) as per the guidelines for detecting these "silent" renal tract abnormalities [27]. The follow-up US examination for persons with high-risk findings, such as trabeculated bladder and HDN, was performed every 6 months or on the onset of any symptoms.

The QOL of a TSCI patient that includes the parameter of a good urological QOL is a significant positive factor in the outcome [28]. The paradigm of comprehensive rehabilitation for a TSCI cohort incorporates an effective bladder management routine to ensure early realization of a good urological QOL. In a Cochrane review on catheter policies for managing long-term voiding problems with neurogenic bladder [29]; no conclusive evidence for dif- ferent catheter management guidelines could be found due to the dynamic nature of the pathophysiology of neurogenic bladder and TSCI. However, an institutional, supervised bladder management protocol is essential. Its effectiveness is highlighted in our study results due to a definitely lower proportion of abnormal findings on renal tract US in our subjects compared to that in subjects from previous studies. We attribute the same to the role of early categorization of our study participants to a particular bladder management routine with supervision and followup with an advantage of the cohort of a patient from the Armed Forces. They are motivated as well as available for follow-up in the rehabilitation center up to 18 months.

Our study has certain limitations, including the relatively small sample size and retrospective design of the study. Further, we did not consider renal function, UTI, pharmacological treatment of bladder dysfunction, and urodynamic findings.

This retrospective pilot study aimed to analyze the incidence of renal tract abnormalities radiologically on US examination alone. Because of the lack of similar studies from our country and especially the military cohort, this study could pave the foundation for future prospective studies with a larger sample size spanning over a longer follow-up duration.

\section{Conclusions}

The present study provides an insight on the incidence of the trabeculated bladder as the most common finding, followed by HDN on routine US of the renal tract in a military cohort of TSCI patients. Those who had sustained an injury 6-12 months previously had fewer renal tract abnormalities, and patients with CIC had a lower prevalence of HDN, attributable to long-term, supervised institutional rehabilitation. There is an association between reflex voiding and trabeculated bladder that indicates the need for further supervision on the amount of abdominal pressure being applied during reflex voiding maneuvers. Patients with no abnormality in US require to be subjected to an annual surveillance scan. At the same time, US findings of bladder trabeculation and HDN merit close follow-up radiologically and clinically to avoid deterioration of renal function and maintain a good urological QOL. 


\section{Conflict of Interest}

No potential conflict of interest relevant to this article was reported.

\section{Acknowledgments}

The authors acknowledge the contribution of Department of Orthopaedics, Military Hospital Kirkee, Pune, India.

\section{Author Contributions}

Study concept: MS, MK, VS, CS; literature search: MS, MK, BG, GSM, CS; manuscript preparation: MS, MK, BG, VS; manuscript editing: MS, BG, VS, GSM; and manuscript review: MS, MK, BG, VS, GSM, CS.

\section{References}

1. Rehabilitation Council of India. Spinal cord injuries [Internet]. New Delhi: Rehabilitation Council of India [cited 2020 Aug 25]. Available from: http://www.rehabcouncil.nic.in/writereaddata/spinal. pdf.

2. Sezer N, Akkus S, Ugurlu FG. Chronic complications of spinal cord injury. World J Orthop 2015;6:24-33.

3. Vellacott PN, Webb-Johnson AE. Spinal injury with retention of urine: the avoidance of catheterisation. Lancet 1919;193:733-7.

4. Bors E, Comarr AE. Mortality. In: Bors E, Comarr AE, editors. Neurological urology: physiology of micturition, its neurological disorders and sequelae. Basel: S. Karger; 1971. p. 351-2.

5. Geisler WO, Jousse AT, Wynne-Jones M, Breithaupt D. Survival in traumatic spinal cord injury. Paraplegia 1983;21:364-73.

6. Strauss DJ, Devivo MJ, Paculdo DR, Shavelle RM. Trends in life expectancy after spinal cord injury. Arch Phys Med Rehabil 2006;87:1079-85.

7. Rabadi MH, Mayanna SK, Vincent AS. Predictors of mortality in veterans with traumatic spinal cord injury. Spinal Cord 2013;51:784-8.

8. Gormley EA. Urologic complications of the neurogenic bladder. Urol Clin North Am 2010;37:601-7.

9. Consortium for Spinal Cord Medicine. Bladder management for adults with spinal cord injury: a clinical practice guideline for health-care providers. J Spinal
Cord Med 2006;29:527-73.

10. Guzelkucuk U, Demir Y, Kesikburun S, Aras B, Yasar E, Tan AK. Ultrasound findings of the urinary tract in patients with spinal cord injury: a study of 1005 cases. Spinal Cord 2015;53:139-44.

11. Edokpolo LU, Foster HE Jr. Renal tract ultrasonography for routine surveillance in spinal cord injury patients. Top Spinal Cord Inj Rehabil 2013;19:54-60.

12. American Spinal Injury Association. Standards for neurological classification of spinal injury patients. Chicago (IL): American Spinal Injury Association; 1982.

13. Taweel WA, Seyam R. Neurogenic bladder in spinal cord injury patients. Res Rep Urol 2015;7:85-99.

14. Gupta S, Chawla JC. Review of urinary tract abnormalities in 100 patients with spinal cord paralysis. Paraplegia 1994;32:531-9.

15. Ku JH, Jung TY, Lee JK, Park WH, Shim HB. Risk factors for urinary stone formation in men with spinal cord injury: a 17-year follow-up study. BJU Int 2006;97:790-3.

16. Hoffberg HJ, Cardenas DD. Bladder trabeculation in spinal cord injury. Arch Phys Med Rehabil 1986;67:750-3.

17. Silva JA, Gonsalves Mde C, de Melo RT, Carrerette FB, Damiao R. Association between the bladder wall thickness and urodynamic findings in patients with spinal cord injury. World J Urol 2015;33:131-5.

18. Pannek J, Bartel P, Gocking K, Frotzler A. Clinical usefulness of ultrasound assessment of detrusor wall thickness in patients with neurogenic lower urinary tract dysfunction due to spinal cord injury: urodynamics made easy? World J Urol 2013;31:659-64.

19. Greenstein A, Rucker KS, Katz PG. Voiding by increased abdominal pressure in male spinal cord injury patients: long term follow up. Paraplegia 1992;30:253-5.

20. Tseng FF, Huang YH, Chen SL, Tsai SJ, Ho CC, Bih LI. Value of Doppler ultrasonography in predicting deteriorating renal function after spinal cord injury. Radiol Med 2012;117:500-6.

21. Hackler RH, Katz PG. Management of common problems in spinal cord injured patients. Am Urol Assoc Update Ser 1991;10:42-7.

22. Pettersson-Hammerstad K, Jonsson O, Svennung IB, Karlsson AK. Impaired renal function in newly spinal cord injured patients improves in the chronic 
state: effect of clean intermittent catheterization? J Urol 2008;180:187-91.

23. $\mathrm{Ku} \mathrm{JH}$, Choi WJ, Lee KY, et al. Complications of the upper urinary tract in patients with spinal cord injury: a long-term follow-up study. Urol Res 2005;33:435-9.

24. Chen Y, DeVivo MJ, Lloyd LK. Bladder stone incidence in persons with spinal cord injury: determinants and trends, 1973-1996. Urology 2001;58:665-70.

25. Ost MC, Lee BR. Urolithiasis in patients with spinal cord injuries: risk factors, management, and outcomes. Curr Opin Urol 2006;16:93-9.

26. Weld KJ, Dmochowski RR. Association of level of injury and bladder behavior in patients with posttraumatic spinal cord injury. Urology 2000;55:490-4.
27. Brandt TD, Neiman HL, Calenoff L, Greenberg M, Kaplan PE, Nanninga JB. Ultrasound evaluation of the urinary system in spinal-cord-injury patients. Radiology 1981;141:473-7.

28. Kumar N, Gupta B. Effect of spinal cord injury on quality of life of affected soldiers in India: a crosssectional study. Asian Spine J 2016;10:267-75.

29. Jamison J, Maguire S, McCann J. Catheter policies for management of long term voiding problems in adults with neurogenic bladder disorders. Cochrane Database Syst Rev 2004;(2):CD004375. 